\title{
Vanishing of doubly symmetrized tensors
}

\author{
Andrew Berget \\ Department of Mathematics \\ University of Washington \\ Seatle, WA, USA \\ aberget@uw.edu
}

\author{
J.A. Dias da Silva Amélia Fonseca \\ Departamento de Matemática \\ Universidade de Lisboa \\ Lisboa, Portugal \\ japsilva@fc.ul.pt, afonseca@ptmat.fc.ul.pt
}

Submitted: Apr 5, 2013; Accepted: Jun 12, 2013; Published: Jun 21, 2013

Mathematics Subject Classifications: 15A69, 05E10, 20C30, 05B35

\begin{abstract}
Symmetrizations of tensors by irreducible characters of the symmetric group serve as natural analogues of symmetric and skew-symmetric tensors. The question of when a symmetrized decomposable tensor is non-zero is intimately related to the rank partition of a matroid extracted from the tensor. In this paper we characterize the non-vanishing of the symmetrization of certain partially symmetrized decomposable tensors. Our answers are phrased in terms of rank partitions of matroids.
\end{abstract}

Keywords: symmetrizations of tensors, matroid, rank partition.

\section{Introduction}

Let $V$ be a finite dimensional complex vector space. Consider the (left) action of the symmetric group $S_{n}$ on the $n$-fold tensor power $V^{\otimes n}$ by permuting tensor factors,

$$
\sigma\left(v_{1} \otimes \cdots \otimes v_{n}\right)=v_{\sigma^{-1}(1)} \otimes \cdots \otimes v_{\sigma^{-1}(n)}
$$

Given a partition $\lambda \vdash n$, let $T_{\lambda} \in \mathbb{C}\left[S_{n}\right]$ denote the projector of the symmetric group algebra $\mathbb{C}\left[S_{n}\right]$ to its isotypic component indexed by $\lambda$.

The image of a decomposable tensor $v^{\otimes}:=v_{1} \otimes \cdots \otimes v_{n}$ under $T_{\lambda}$ is called a symmetrized decomposable tensor. These are closely related to irreducible character immanants of positive semi-definite matrices [7, Chapters 6-7]. A fundamental question is when a symmetrized decomposable tensor is zero. This question was first answered by Gamas [4] and later characterized in terms of the matroid of the vectors $v_{1}, \ldots, v_{n}$, as we now describe.

We will let $v=\left(v_{1}, \ldots, v_{n}\right)$ denote a configuration of $n$ vectors in $V$, and $M(v)$ the corresponding matroid. Given any matroid $M$, the rank partition of $M$ is the sequence of numbers $\rho(M)=\left(\rho_{1}, \rho_{2}, \ldots\right)$ determined by the condition that its $k$ th partial sum is the size of the largest union of $k$ independent sets of $M$. 
Theorem 1 ([2, Theorem $\left.\left.\mathrm{A}^{\prime}\right]\right)$. The symmetrized decomposable tensor $T_{\lambda} v^{\otimes}$ is non-zero if and only if $\lambda^{t} \leqslant \rho(M(v))$.

Here, $\lambda^{t}$ denotes the conjugate partition of $\lambda$, and $\leqslant$ denotes dominance order on the set of partitions of size $n$.

The main result of the current paper is a generalization of this result, where one first partially symmetrizes $v^{\otimes}$.

Theorem 2. Let $\lambda$ and $\mu$ be partitions of $n$.

(1) Then there is a permutation $\pi \in S_{n}$ such that

$$
T_{\lambda}\left(\left(v_{\pi(1)} \wedge \cdots \wedge v_{\pi\left(\mu_{1}\right)}\right) \otimes \cdots \otimes\left(v_{\pi\left(n-\mu_{r}+1\right)} \wedge \cdots \wedge v_{\pi(n)}\right)\right) \neq 0
$$

if and only if $\mu \leqslant \lambda^{t} \leqslant \rho(M(v))$.

(2) Dually, there is a permutation $\pi \in S_{n}$ such that

$$
T_{\lambda}\left(\left(v_{\pi(1)} \cdots v_{\pi\left(\mu_{1}\right)}\right) \otimes \cdots \otimes\left(v_{\pi\left(n-\mu_{r}+1\right)} \cdots v_{\pi(n)}\right)\right) \neq 0
$$

if and only if $\mu \leqslant \lambda$ and $\lambda^{t} \leqslant \rho(M(v))$.

Here we are viewing the tensor product of the exterior and symmetric powers of $V$ as subspaces of $V^{\otimes n}$.

The outline of this paper is as follows: We first present some background on representations of the symmetric group and matroids. Then we consider the notion of compatible pairs of tableaux. With these preliminaries, we next prove Theorem 2. We end with a brief discussion of a conjecture that motivated the theorem.

\section{Background}

In this section we review some relevant notions from representation theory, and matroid theory.

\subsection{Representation theory}

We refer to the book of James and Kerber [5] for a thorough treatment of the representation theory of the symmetric group.

The group of permutations of the set $[n]:=\{1,2, \ldots, n\}$ is denoted $S_{n}$. The irreducible representations of $S_{n}$ are parametrized by partitions $\lambda=\left(\lambda_{1} \geqslant \lambda_{2} \geqslant \ldots \lambda_{\ell} \geqslant 0\right)$ of $n$; $\sum_{i} \lambda_{i}=n$. We construct these representations as follows.

A tableau is a filling $T$ of the Young diagram of $\lambda$ with the numbers in $[n]$, each number being used exactly once. A tableau is said to be standard if the numbers in the 
filling increase across rows and columns. Given a tableau $T$, define two elements of the group algebra $\mathbb{C}\left[S_{n}\right]$ by

$$
p_{T}=\sum_{\sigma \in \operatorname{Row}(T)} \sigma, \quad n_{T}=\sum_{\sigma \in \operatorname{Col}(T)} \operatorname{sign}(\sigma) \sigma .
$$

Here, $\operatorname{Row}(T)$ is the group of permutations that take each row of $T$ into itself, and $\operatorname{Col}(T)$ is likewise defined for the columns of $T$. These elements are, respectively, the row symmetrizer and column anti-symmetrizer of $T$.

The product $p_{T} n_{T}$ is called a Young symmetrizer, and we will call the product $n_{T} p_{T}$ a dual Young symmetrizer. It is a theorem that the left ideal in $\mathbb{C}\left[S_{n}\right]$ generated by a (dual) Young symmetrizer is an irreducible representations of $S_{n}$. Further, if $S$ and $T$ are two tableaux, then the representations generated by $p_{S} n_{S}$ and $p_{T} n_{T}$ are isomorphic if and only if the shape of $S$ equals the shape of $T$. We say that an irreducible representation of $S_{n}$ has shape $\lambda$ if it is isomorphic to the ideal generated by some $p_{T} n_{T}$ for a tableau $T$ of shape $\lambda$. It is a fact that $p_{T} n_{T}$ and $n_{T} p_{T}$ generate isomorphic representations.

The projection of $\mathbb{C}\left[S_{n}\right]$ onto its isotypic component of shape $\lambda$ is denoted $T_{\lambda}$. It is fact that

$$
T_{\lambda}=\frac{\chi^{\lambda}(1)}{n !} \sum_{\sigma \in S_{n}} \chi(\sigma) \sigma \in \mathbb{C}\left[S_{n}\right],
$$

and that $T_{\lambda}$ is a scalar multiple of $\sum_{T} p_{T} n_{T}=\sum_{T} n_{T} p_{T}$, the sum over all tableaux of shape $\lambda$. Indeed, $P_{\lambda}:=\sum_{T} p_{T} n_{T}$ is central since $\sigma p_{T} n_{T} \sigma^{-1}=p_{\sigma T} n_{\sigma T}$, and hence Schur's lemma implies that $P_{\lambda}$ acts on each irreducible representation of $S_{n}$ by a scalar determined only by the isomorphism type. Since $P_{\lambda}$ annihilates Young symmetrizers of shapes other than $\lambda$ and it is non-zero in the group algebra, it must act on an irreducible of shape $\lambda$ by a non-zero scalar. In particular, this means $P_{\lambda}$ is essentially idempotent. Similar reasoning shows that $\sum_{T} p_{T} n_{T}$ satisfies these properties too.

Let $V$ be a finite dimensional complex vector space. The symmetric group $S_{n}$ acts on the left of $V^{\otimes n}$ via permutation of tensor factors, as in the introduction. We conclude that $T_{\lambda}$ acts on $V^{\otimes n}$ as projection to its isotypic component of shape $\lambda$; the image of $T_{\lambda}$ is referred to as a symmetry class of tensors of shape $\lambda$. Such tensors are important from the point of view of representation theory of the general linear group, invariant theory and algebraic geometry [3, Chapters 8-9].

\subsection{Matroid theory}

Given a finite collection of vectors $v_{1}, \ldots, v_{n}$ selected from a fixed vector space, we construct a simplicial complex $M(v)$ as follows. The vertex set of $M(v)$ is $[n]$ and the faces of $M(v)$ are those subsets $I \subset[n]$ such that $\left\{v_{i}: i \in I\right\}$ is a linearly independent set. $M(v)$ is the matroid of $v$. The faces of $M(v)$ are referred to as independent sets, while non-faces are said to be dependent.

The matroid of $v$ is an example of a matroid complex (or simply, matroid). These are simplicial complexes whose faces satisfy the exchange axiom: If $I$ and $I^{\prime}$ are faces and 
$|I|<\left|I^{\prime}\right|$, then there is $e \in I^{\prime} \backslash I$ such that $I \cup\{e\}$ is a still a face. We will assume from now on that all matroids considered are loopless, meaning that there are no singleton dependent sets, in particular, $v_{i} \neq 0$ for all $i$.

Given a matroid $M$ with ground set $[n]$ and a partition $\lambda$ of $n$, we define a $\lambda$-coloring of $M$ to be a set partition of $[n]$ into blocks of size $\lambda_{1}, \lambda_{2}, \ldots$ each of which is an independent set of $M$. Thus, $M$ is $\lambda$-colorable if there is a tableau of shape $\lambda$ whose rows index independent sets of $M$.

As in the introduction, the rank partition of $M, \rho(M)$, is defined by the condition that the $k$ th partial sum of its parts is equal to the size of the largest union of $k$ independent sets of $M$. In [2] it is proved that $\rho(M)$ is actually a partition of $M$. Further, it is shown that $M$ is $\lambda$-colorable if and only if $\lambda \leqslant \rho(M)$.

The length of the rank partition appears in the graph theory literature under the guise of arboricity. Recently, the third author has studied the rank partition in the context of multi-graded Hilbert series of projective equivalence classes.

\section{Compatible pairs of tableaux}

In this section we define the notion of a compatible pair of tableaux. We then investigate the combinatorics of such pairs relevant to the proof of Theorem 2 .

For each tableau $T$ of shape $\lambda$, we define a function $c_{T}:[n] \rightarrow\left[\lambda_{1}\right]$ by the rule $c_{T}(i)=$ the column number of $T$ that contains $i$. We say that a pair of tableaux $(S, T)$ is compatible if whenever $i$ and $j$ are in the same row of $S, c_{S}(i)<c_{S}(j)$ implies $c_{T}(i)<c_{T}(j)$. It is clear that if $(S, T)$ is a compatible pair, then $\operatorname{Row}(S) \cap \operatorname{Col}(T)=\{1\}$. The converse is false, but we offer the following in lieu of it.

Proposition 3. Let $(S, T)$ be a pair of tableaux such that $\operatorname{Row}(S) \cap \operatorname{Col}(T)=\{1\}$. Then, there exists $\pi \in \operatorname{Row}(S)$ such that $(\pi S, T)$ is a compatible pair of tableaux.

Proof. The hypothesis implies that any two numbers $i, j$ that occur in the same row of $S$ occur in different columns of $T$. Denote the entries in the $i$ th row (in order) of $S$ by $s_{1}, \ldots, s_{r}$ and rearrange them to $s_{1}^{\prime}, \ldots, s_{r}^{\prime}$ so that $c_{T}\left(s_{j}^{\prime}\right)<c_{T}\left(s_{j+1}^{\prime}\right)$. Doing this procedure for each row proves the proposition.

Proposition 4. Let $(S, T)$ be a compatible pair of tableaux. Suppose that $\tau \in \operatorname{Col}(S)$, $\sigma \in \operatorname{Row}(S)$ and $\tau \sigma \in \operatorname{Col}(T)$. Then, we must have $\sigma=1$.

We will employ the following notation in the proof: If $T$ is a tableau, then $K_{i}(T)$ denotes the set of entries in column $i$ of $T$.

Proof. For $\sigma \in S_{n}$ let $\operatorname{Fix}(\sigma)$ denote its set of fixed points. Say that $S$ and $T$ are tableaux of shape $\lambda$ and $\mu$, respectively.

Let $\tau \in \operatorname{Col}(S), \sigma \in \operatorname{Row}(S)$ and $s \in\left\{1, \ldots, \mu_{1}\right\}$. We will prove by induction on $s$ that if, for $i=1, \ldots, s$,

$$
\tau \sigma\left(K_{i}(T)\right)=K_{i}(T)
$$


then

$$
\bigcup_{i=1}^{s} K_{i}(T) \subseteq \operatorname{Fix}(\sigma) .
$$

Suppose that $\tau \sigma\left(K_{1}(T)\right)=K_{1}(T)$. Observe that $K_{1}(T) \subseteq K_{1}(S)$, since $(S, T)$ is a compatible pair of tableaux. Let $a \in K_{1}(T)$. Then $\tau \sigma(a) \in K_{1}(T)$ and hence $a, \tau \sigma(a) \in$ $K_{1}(S)$. Let $t$ be such that $\sigma(a) \in K_{t}(S)$. Since $\tau \in \operatorname{Col}(S)$, we have that $\tau \sigma(a) \in K_{t}(S)$. Therefore $t=1$ and hence $a, \sigma(a) \in K_{1}(S)$. Since $\sigma \in \operatorname{Row}(S)$, we have $\sigma(a)=a$.

Assume that $s>1$ and that $\tau \sigma\left(K_{i}(T)\right)=K_{i}(T)$, for every $i=1, \ldots, s$. By the induction hypothesis, $\bigcup_{t=1}^{s-1} K_{t}(T) \subseteq \operatorname{Fix}(\sigma)$. It remains to prove that $K_{s}(T) \subseteq \operatorname{Fix}(\sigma)$.

Let $H_{i}=K_{s}(T) \cap K_{i}(S)$, for $i=1, \ldots, \lambda_{1}$. Then $K_{s}(T)=\bigcup_{i=1}^{\lambda_{1}} H_{i}$.

Suppose that $K_{s}(T) \nsubseteq \operatorname{Fix}(\sigma)$. Let $p=\max \left\{i: H_{i} \nsubseteq \operatorname{Fix}(\sigma)\right\}$. Choose $a \in H_{p}$ such that $\sigma(a) \neq a$. As $\sigma \in \operatorname{Row}(S), a \in K_{p}(S)$, and $\sigma(a) \neq a$, we have $\sigma(a) \notin K_{p}(S)$. Let $q$ such that $\sigma(a) \in K_{q}(S)$. We have $q \neq p$. Since $\tau \in \operatorname{Col}(S), \tau \sigma(a) \in K_{q}(S)$. As $a \in H_{p}$, $a \in K_{s}(T)$, thus $\tau \sigma(a) \in K_{s}(T)$. Therefore $\tau \sigma(a) \in H_{q}$. We consider two cases.

Case 1. Assume that $q<p$. Since $\sigma \in \operatorname{Row}(S), a$ and $\sigma(a)$ are in the same row of $S$. We have $\sigma(a) \in K_{q}(S), a \in K_{p}(S)$ and $q<p$. Since $a \in K_{s}(T)$ and $(S, T)$ is a compatible pair of tableaux, $\sigma(a) \in \bigcup_{t=1}^{s-1} K_{t}(T) \subseteq \operatorname{Fix}(\sigma)$. Then $\sigma(\sigma(a))=\sigma(a)$. This is a contradiction because $\sigma(a) \neq a$.

Case 2. Assume that $p<q$. By the definition of $p, H_{q} \subseteq \operatorname{Fix}(\sigma)$. We claim that $\tau \sigma\left(H_{q}\right)=$ $H_{q}$. Indeed, let $b \in H_{q}$. Then $\sigma(b)=b \in K_{q}(S)$ therefore, since $\tau \in \operatorname{Col}(S)$, $\tau \sigma(b) \in K_{q}(S)$; as $b \in K_{s}(T), \tau \sigma(b) \in K_{s}(T)$, hence $\tau \sigma(b) \in K_{s}(T) \cap K_{q}(S)=H_{q}$. We proved that $\tau \sigma\left(H_{q}\right) \subseteq H_{q}$. Since $\tau \sigma$ is one to one, $\tau \sigma\left(H_{q}\right)=H_{q}$ and we get a contradiction because $a \notin H_{q}$ and $\tau \sigma(a) \in H_{q}$.

Now if $\tau \in \operatorname{Col}(S), \sigma \in \operatorname{Row}(S)$ and $\tau \sigma \in \operatorname{Col}(S)$, then

$$
\tau \sigma\left(K_{i}(T)\right)=K_{i}(T),
$$

for $i=1, \ldots, \mu_{1}$. Therefore

$$
\bigcup_{t=1}^{\mu_{1}} K_{t}(T) \subseteq \operatorname{Fix}(\sigma)
$$

and we have $\sigma=1$.

Lemma 5. If $(S, T)$ is a compatible pair of tableaux, then

$$
p_{S} n_{T} n_{S}=|\operatorname{Col}(S) \cap \operatorname{Col}(T)| p_{S} n_{S} .
$$

Proof. We consider the coefficient of the identity element 1 in the product $p_{S} n_{T} n_{S}$. It is given by the formula,

$$
\sum_{\substack{\sigma \in \operatorname{Row}(S), \tau \in \operatorname{Col}(T), \nu \in \operatorname{Col}(S) \\ \sigma \tau \nu=1}} \operatorname{sign}(\tau) \operatorname{sign}(\nu) .
$$


If $\sigma \in \operatorname{Row}(S), \tau \in \operatorname{Col}(T), \nu \in \operatorname{Col}(S)$ and $\sigma \tau \nu=1$, then $\tau=\sigma^{-1} \nu^{-1} \in \operatorname{Col}(T)$, and, by Proposition $4, \sigma=1$ and $\tau=\nu^{-1}$. Therefore, the above sum reduces to being over $|\operatorname{Col}(S) \cap \operatorname{Col}(T)|$, and each summand reduces to 1 .

For every $\sigma \in \operatorname{Row}(S)$ and every $\tau \in \operatorname{Col}(S)$,

$$
\sigma \cdot p_{S} n_{T} n_{S} \cdot \tau=\operatorname{sign}(\tau) p_{S} n_{T} n_{S}
$$

We conclude from $[6, p p .501-503]$ that $p_{S} n_{T} n_{S}=|\operatorname{Col}(S) \cap \operatorname{Col}(T)| p_{S} n_{S}$.

From the lemma we at once obtain a dual version.

Corollary 6. If $\left(S^{t}, T^{t}\right)$ is a compatible pair of tableaux, then

$$
n_{S} p_{T} p_{S}=|\operatorname{Row}(S) \cap \operatorname{Row}(T)| n_{S} p_{S}
$$

Proof. The symmetric group algebra $\mathbb{C}\left[S_{n}\right]$ has an involutive automorphism $\iota$ induced by the map $\sigma \mapsto \operatorname{sign}(\sigma) \sigma$. This follows since sign is a linear character of $S_{n}$. This map has

the property that $\iota p_{S}=n_{S^{t}}$ and $\iota n_{S}=p_{S^{t}}$. Applying $\iota$ to the result of Lemma 5 gives the claimed equality.

\section{Proof of Theorem 2}

We restate our main theorem.

Theorem 2. Let $\lambda$ and $\mu$ be partitions of $n$.

(1) Then there is a permutation $\pi \in S_{n}$ such that

$$
T_{\lambda}\left(\left(v_{\pi(1)} \wedge \cdots \wedge v_{\pi\left(\mu_{1}\right)}\right) \otimes \cdots \otimes\left(v_{\pi\left(n-\mu_{r}+1\right)} \wedge \cdots \wedge v_{\pi(n)}\right)\right) \neq 0
$$

if and only if $\mu \leqslant \lambda^{t} \leqslant \rho(M(v))$.

(2) Dually, there is a permutation $\pi \in S_{n}$ such that

$$
T_{\lambda}\left(\left(v_{\pi(1)} \cdots v_{\pi\left(\mu_{1}\right)}\right) \otimes \cdots \otimes\left(v_{\pi\left(n-\mu_{r}+1\right)} \cdots v_{\pi(n)}\right)\right) \neq 0
$$

if and only if $\mu \leqslant \lambda$ and $\lambda^{t} \leqslant \rho(M(v))$.

Proof of Theorem 2(1). We start by assuming that the tensor occuring in (1) is non-zero. The tensor,

$$
\left(v_{\pi(1)} \wedge \cdots \wedge v_{\pi\left(\mu_{1}\right)}\right) \otimes \cdots \otimes\left(v_{\pi\left(n-\mu_{r}+1\right)} \wedge \cdots \wedge v_{\pi(n)}\right)
$$

is obtained by applying a column anti-symmetrizer $n_{T}$ to $v_{1} \otimes \cdots \otimes v_{n}$, for some tableau $T$ of shape $\mu^{t}$. Since $T_{\lambda} \in \mathbb{C}\left[S_{n}\right]$ is central, we have

$$
T_{\lambda} n_{T}\left(v_{1} \otimes \cdots \otimes v_{n}\right)=n_{T} T_{\lambda}\left(v_{1} \otimes \cdots \otimes v_{n}\right) \neq 0 .
$$

In particular, $T_{\lambda}\left(v_{1} \otimes \cdots \otimes v_{n}\right) \neq 0$. By Theorem 1 , we know that $\lambda^{t} \leqslant \rho(M(v))$. 
Now, the Frobenius character of the left ideal in $\mathbb{C}\left[S_{n}\right]$ generated by $n_{T}$ is the elementary symmetric function $e_{\mu}$. The fact that $n_{T} T_{\lambda} \neq 0$ implies that in the Schur expansion of $e_{\mu}$, the Schur function $s_{\lambda}$ appears with positive coefficient. However, this coefficient is the Kostka number $K_{\lambda^{t} \mu}$ and hence $\lambda^{t} \geqslant \mu$.

For the opposite direction, we assume that $\mu \leqslant \lambda^{t} \leqslant \rho(M(v))$. It follows that $\lambda \leqslant \mu^{t}$, and by [5, Lemma 1.4.20] there is a pair of tableaux $\left(S_{0}, T\right)$ of shape $\lambda$ and $\mu^{t}$, respectively, such that any two elements in the same row of $S_{0}$ occur in the different columns of $T$. From this we have $\operatorname{Row}\left(S_{0}\right) \cap \operatorname{Col}(T)=\{1\}$ and by Proposition 3, there is some $\pi \in \operatorname{Row}\left(S_{0}\right)$ such that $\left(\pi S_{0}, T\right)$ is a compatible pair of tableaux. Write $S=\pi S_{0}$.

Since $\lambda^{t} \leqslant \rho(M(v))$, Theorem 1 gives us $T_{\lambda} v^{\otimes} \neq 0$. Since $T_{\lambda}$ is scalar multiple of $\sum_{Q} p_{Q} n_{Q}$ (the sum over all tableaux $Q$ of shape $\lambda$ ) there is some summand $p_{Q} n_{Q}$ which is not zero when applied to $v^{\otimes}$. By rearranging the vectors we may assume that this summand is $p_{S} n_{S} v^{\otimes}$.

Lemma 5 tells us that $p_{S} n_{T} n_{S}=|\operatorname{Col}(S) \cap \operatorname{Col}(T)| p_{S} n_{S}$. A Young symmetrizer $p_{S} n_{S}$ is essentially idempotent, thus, there is a non-zero constant $k$ for which

$$
k \cdot p_{S} n_{S}=\left(p_{S} n_{S}\right)\left(p_{S} n_{T} n_{S}\right) .
$$

Applying $k \cdot p_{S} n_{S}$ to $v_{1} \otimes \cdots \otimes v_{n}$ we see that

$$
\left(n_{S} p_{S} n_{T} n_{S}\right)\left(v_{1} \otimes \cdots \otimes v_{n}\right) \neq 0
$$

and this implies that there is some permutation $\sigma \in \operatorname{Col}(S)$ for which,

$$
\left(n_{S} p_{S} n_{T}\right) v_{\sigma^{-1}(1)} \otimes \cdots \otimes v_{\sigma^{-1}(n)} \neq 0 .
$$

This implies that the cyclic $S_{n}$-representation in $V^{\otimes n}$ generated by

$$
n_{T}\left(v_{\sigma^{-1}(1)} \otimes \cdots \otimes v_{\sigma^{-1}(n)}\right)
$$

contains an irreducible representation of shape $\lambda$, since $n_{S} p_{S}$ is a dual Young symmetrizer. We conclude the projection of this cyclic representation to its isotypic component of shape $\lambda$ is non-zero, and hence,

$$
T_{\lambda} n_{T}\left(v_{\sigma^{-1}(1)} \otimes \cdots \otimes v_{\sigma^{-1}(n)}\right) \neq 0 .
$$

Reindexing the vectors if needed, this is exactly what we needed to show.

We now prove the version for symmetrizations of products of symmetric tensors, the proof following exactly the same lines as that of (1).

Proof of Theorem 2(2). Assume that the tensor occuring in (2) is non-zero. The hypothesis implies that there is a tableau $T$ of shape $\mu$ for which $T_{\lambda} p_{T} v^{\otimes} \neq 0$. This implies $T_{\lambda} p_{T} \neq 0$ and, as before, this implies the Schur function $s_{\lambda}$ appears in the Schur decomposition of $h_{\lambda}$. Since this is the Kostka number $K_{\lambda \mu}$, we must have $\mu \leqslant \lambda$. As before, $T_{\lambda} p_{T}=p_{T} T_{\lambda}$, and thus $T_{\lambda} v^{\otimes} \neq 0$, forcing $\lambda^{t} \leqslant \rho(M(v))$. 
For the other direction we assume that $\mu \leqslant \lambda$ and that $\lambda^{t} \leqslant \rho(M(v))$. The former hypothesis furnishes us with a compatible pair of tableaux $\left(S^{t}, T^{t}\right)$ of shape $\lambda^{t}$ and $\mu^{t}$, respectively. The latter hypothesis implies that $T_{\lambda} v^{\otimes} \neq 0$.

Since $T_{\lambda}$ is a non-zero scalar multiple of $\sum_{Q} n_{Q} p_{Q}$ (sum over tableaux of shape $\lambda$ ), there is a dual Young symmetrizer $n_{Q} p_{Q}$ for which $n_{Q} p_{Q} v^{\otimes} \neq 0$. We may reindex $v$ so that $Q=S$.

Now, we apply Corollary 6 and the fact that dual Young symmetrizers are near idempotent to get that

$$
\left(n_{S} p_{S}\right)\left(n_{S} p_{T} p_{S}\right) v^{\otimes} \neq 0 .
$$

From this we conclude that there is some permutation $\sigma \in \operatorname{Row}(S)$ for which

$$
p_{S} n_{S} p_{T} v_{\sigma^{-1}(1)} \otimes \cdots \otimes v_{\sigma^{-1}(n)} \neq 0 .
$$

Just as in the proof of (1), this statement is sufficient to give the claim of the theorem.

\section{$5 \quad$ Representations generated by tensors}

We briefly mention the representation theory that motivated our theorem.

The general linear group $\mathrm{GL}(V)$ acts diagonally on the tensor product $V^{\otimes n}$. Given $x \in V^{\otimes n}$ we let $G(x)$ denote the cyclic GL(V)-representation in $V^{\otimes n}$ generated by $x$. That is, $G(x)$ is linearly spanned by the orbit $\mathrm{GL}(V) x$.

It follows from Schur-Weyl duality that an irreducible representation of $\mathrm{GL}(V)$ with highest weight $\lambda$ appears in $G(x)$ if and only if $T_{\lambda} x \neq 0$. Applying Theorem 1 we see that the support of the irreducible decomposition of $G\left(v^{\otimes}\right)$ is an invariant of $M(v)$. The third author (in joint work with A. Fink) has recently shown [1] that the isomorphism type of $G\left(v^{\otimes}\right)$ is an invariant of $M(v)$.

The character of $G\left(v^{\otimes}\right)$ is a symmetric polynomial in $\operatorname{dim}(V)$-many variables, and is a positive integer sum of Schur polynomials. For $\lambda^{t} \leqslant \rho(M(v))$, it has been observed through computer experimentation that the difference between the character of $G\left(v^{\otimes}\right)$ and the complete homogeneous symmetric polynomial $h_{\lambda}$ remains a positive integer sum of Schur polynomials.

This naturally leads a person to look for surjections from $G\left(v^{\otimes}\right)$ to representations whose character is $h_{\lambda}$. From this we arrive at the following conjecture.

Conjecture 7. Suppose that $\mu^{t} \leqslant \rho(M(v))$, and let $S$ denote the tableau of shape $\mu$ filled with the numbers $1, \ldots, n$ from left-to-right, top-to-bottom. Then, there is a permutation $\sigma \in S_{n}$ such that the natural map,

$$
p_{S} \sigma: G\left(v^{\otimes}\right) \rightarrow \operatorname{Sym}^{\mu_{1}}(V) \otimes \cdots \otimes \operatorname{Sym}^{\mu_{r}}(V),
$$

is surjective.

The conjecture would imply that $G\left(p_{S} \sigma v^{\otimes}\right)$ contains an irreducible representation of shape $\lambda$ for every $\lambda \geqslant \mu$. The second item in our Theorem 2 is a weakening of this assertion. 
This conjecture is best possible, in the following sense. Let

$$
v^{\otimes}=\mathbf{e}_{\mu}^{\otimes}=\underbrace{\left(\mathbf{e}_{1} \otimes \cdots \otimes \mathbf{e}_{1}\right)}_{\mu_{1}} \otimes \cdots \otimes \underbrace{\left(\mathbf{e}_{r} \otimes \cdots \otimes \mathbf{e}_{r}\right)}_{\mu_{r}},
$$

where $\mathbf{e}_{i}$ denotes the $i$ th standard basis vector of $V=\mathbb{C}^{r}$. In this case $G\left(\mathbf{e}_{\mu}^{\otimes}\right)$ actually is a tensor product of symmetric powers, and it is readily shown that the conjecture holds at the level of characters. Indeed it is sufficient to prove it when $\lambda \geqslant \mu$ is a covering relation. In this case $\lambda$ and $\mu$ differ in exactly two positions, and it is sufficient to see that $h_{a} h_{b}-h_{a+1} h_{b-1}$ is Schur positive, where $a \geqslant b \geqslant 1$. This follows from the Pieri rule since the difference is exactly the Schur polynomial $s_{(a, b)}$.

An interesting combinatorial problem related to Conjecture 7 is to put a lower bound on the number of distinct $\lambda$-colorings of a matroid, given that $\lambda \leqslant \rho(M)$.

\section{References}

[1] A. Berget, A. Fink, Equivariant $K$-classes of matrix orbit closures, arXiv:1306.1810, 2013.

[2] J. A. Dias da Silva, On the $\mu$-colorings of a matroid, Linear and Multilinear Algebra 27, 25-32 (1990).

[3] W. Fulton, Young tableaux, With applications to representation theory and geometry, London Math. Soc. Student Texts, vol. 35, Cambridge University Press, Cambridge, 1997.

[4] C. Gamas, Conditions for a symmetrized decomposable tensor to be zero, Linear Algebra Appl. 108, 83-119 (1988).

[5] G. James and A. Kerber, The Representation Theory of the Symmetric Group, Addison Wesley, Reading MA, 1981.

[6] M. Marcus, Finite Dimensional Multilinear Algebra, Part II, Marcel Dekker, New York, 1975.

[7] R. Merris, Multilinear Algebra, Gordon and Breach Science Publishers, 1997. 